\title{
Association between periodontitis and prognosis of pancreatobiliary tract cancer: A pilot study
}

\author{
TAKAYUKI MARUYAMA ${ }^{1}$, TAKAAKI TOMOFUJI ${ }^{2}$, TATSUYA MACHIDA ${ }^{3}$, HIRONARI KATO $^{4}$, \\ KOICHIRO TSUTSUMI ${ }^{4}$, DAISUKE UCHIDA ${ }^{4}$, AKINOBU TAKAKI ${ }^{4}$, TOSHIKI YONEDA $^{3}$, \\ HISATAKA MIYAI $^{3}$, HIROFUMI MIZUNO ${ }^{3}$, DAISUKE EKUNI ${ }^{3}$, HIROYUKI OKADA $^{4}$ and MANABU MORITA ${ }^{3}$ \\ ${ }^{1}$ Center for Innovative Clinical Medicine, Okayama University Hospital, Okayama 700-8558; \\ ${ }^{2}$ Department of Community Oral Health, Asahi University School of Dentistry, Mizuho, Gifu 501-0296; \\ ${ }^{3}$ Department of Preventive Dentistry, Okayama University Graduate School of Medicine, Dentistry \\ and Pharmaceutical Sciences; ${ }^{4}$ Department of Gastroenterology and Hepatology, Okayama University \\ Graduate School of Medicine, Dentistry and Pharmaceutical Sciences, Okayama 700-8558, Japan
}

Received July 25, 2016; Accepted March 10, 2017

DOI: $10.3892 / \mathrm{mco} .2017 .1220$

\begin{abstract}
Several studies have indicated that periodontitis is a risk factor for cancer. However, the association between periodontitis and the prognosis of pancreatobiliary tract cancer remains unclear. The aim of this pilot study was to investigate the association between periodontitis and prognosis of pancreatobiliary tract cancer. A total of 22 patients diagnosed with pancreatobiliary tract cancer were analyzed. Oral health status, including severity of periodontitis, general health status and biochemical serum markers were evaluated. The Kaplan-Meier method and Cox proportional hazards model were used to assess factors affecting the prognosis of pancreatobiliary tract cancer. The Kaplan-Meier analysis demonstrated that low body mass index, high concentration of serum C-reactive protein (CRP) and severe periodontitis were significant prognostic factors for survival rate. The Cox proportional hazards model revealed that serum carbohydrate antigen 19-9 concentration [hazard ratio $(\mathrm{HR})=1.002$; 95\% confidence interval (CI): 1.000-1.004] and serum CRP concentration $(\mathrm{HR}=2.57$; 95\% CI: 1.15-5.74) were significantly associated with the prognosis of pancreatobiliary tract cancer. In addition, cancer patients with severe periodontitis
\end{abstract}

Correspondence to: Dr Takayuki Maruyama, Center for Innovative Clinical Medicine, Okayama University Hospital, 2-5-1 Shikata-cho, Kita-ku, Okayama 700-8558, Japan

E-mail: t-maru@md.okayama-u.ac.jp

Abbreviations: BMI, body mass index; \%BOP, percentage of sites with bleeding on probing; CA19-9, carbohydrate antigen 19-9; CAL, clinical attachment level; CEA, carcinoembryonic antigen; CI, confidence interval; CRP, C-reactive protein; HbA1c, hemoglobin A1c; HR, hazard ratio; PPD, probing pocket depth

Key words: periodontitis, pancreatobiliary tract cancer, prognosis, epidemiology had higher serum CRP concentrations compared with those without severe periodontitis. Therefore, severe periodontitis indirectly affected the prognosis of pancreatobiliary tract cancer through promoting systemic inflammation.

\section{Introduction}

Pancreatobiliary tract cancer is a general term for malignancies in the pancreas, gallbladder and extrahepatic bile ducts. Despite advances in cancer treatment, the prognosis of pancreatobiliary tract cancer remains very poor $(1,2)$. According to the American Cancer Society (3), for all stages of pancreatic cancer combined, the 1-year relative survival rate is $20 \%$, and the 5 -year rate is $6 \%$. The risk factors for pancreatic cancer include old age, male gender, smoking, chronic pancreatitis, type 2 diabetes mellitus, obesity and a family history of pancreatic cancer (4). However, the prognostic factors for pancreatic cancer remain unclear. Furthermore, possibly due to its infrequent occurrence and rapidly fatal outcome, little clinical research has been performed on the etiology of gallbladder and extrahepatic bile duct cancer. Therefore, it is crucial to investigate factors affecting the survival rate of pancreatobiliary tract cancer.

It has been reported that periodontitis is associated with numerous systemic diseases, including cancer (5). Periodontitis is a chronic inflammatory disease of the tissues that support the teeth, and it is initiated by overgrowth of anaerobic bacteria in subgingival sites. Development of periodontitis depends on the interaction between bacterial products, such as lipopolysaccharides, and the host, leading to cytokine production that mediates periodontal tissue destruction. Periodontal infections are associated with elevated concentrations of circulating inflammatory cytokines $(6,7)$ and the severity of periodontitis is directly correlated with serum concentrations of inflammatory cytokines (8). Upregulation of inflammatory cytokine pathways have been shown to modulate pancreatic cancer progression (9). These studies suggest that periodontitis may affect the progression as well as the prognosis of pancreatobiliary tract cancer. 
Previous studies have indicated that periodontitis is a risk factor for pancreatic cancer $(10,11)$. However, the association between periodontitis and the prognosis of pancreatobiliary tract cancer remains unclear, but it has been hypothesized that severe periodontitis may affect the prognosis of pancreatobiliary tract cancer. The aim of this pilot study was to investigate the association between periodontitis and the prognosis of pancreatobiliary tract cancer.

\section{Patients and methods}

Study population. A total of 77 patients were diagnosed with primary cancer of the pancreas, bile ducts or gallbladder at the Department of Gastroenterology and Hepatology, Okayama University Hospital (Okayama, Japan), between July, 2013 and July, 2014. Among those patients, 30 (20 men and 10 women) were referred to the Department of Preventive Dentistry for oral health checks prior to cancer therapy. Patients who were aged $<50$ years ( 1 patient), who had $<10$ teeth ( 4 patients), whose 1-year survival data were missing ( 2 patients) and who lacked data (1 patient) were excluded from this study. As a result, data from 22 patients (14 men and 8 women) were analyzed. This study was approved by the Ethics Committee of the Okayama University Graduate School of Medicine, Dentistry and Pharmaceutical Sciences and the Okayama University Hospital (no. 1506-052). Written informed consent was obtained from all patients who agreed to participate.

Oral examination. One dentist recorded the following data: Number of teeth present, probing pocket depth (PPD), clinical attachment level (CAL), percentage of sites with bleeding on probing $(\% \mathrm{BOP})$ and percentage of sites with plaque accumulation. PPD and CAL were measured for all teeth present, except for third molars, using a color-coded probe (CP-8, Hu-Friedy, Chicago, IL, USA). CAL was measured as the distance between the cemento-enamel junction and the base of the periodontal pocket. PPD and CAL were measured at six sites (mesio-buccal, mid-buccal, disto-buccal, mesio-lingual, mid-lingual and disto-lingual). BOP was defined as the presence of bleeding after gentle probing with $25 \mathrm{~g}$ probing force. Plaque level was measured after staining with erythrosine and was recorded as the presence or absence of plaque at four sites (mesial, distal, buccal and lingual) around each tooth. Mean PPD and CAL, \%BOP and plaque level were calculated for each patient. In addition, the severity of periodontitis (no, mild, moderate or severe periodontitis) for each patient was defined as previously described (12).

General status examination. Prior to cancer therapy, medical charts were reviewed to obtain information on cancer site, cancer stage, body weight and height. Body mass index (BMI) was calculated using body weight and height data. A personal interview was conducted to obtain information on smoking habits (pack-years). In addition, biochemical serum markers, including hemoglobin A1c (HbA1c), C-reactive protein (CRP), albumin, carcinoembryonic antigen (CEA) and carbohydrate antigen 19-9 (CA19-9) were also evaluated. These serum markers are commonly assessed in the prognosis of pancreatobiliary tract cancer (13). Serum HbA1c concentration was measured by high-performance liquid chromatography. Serum
CRP concentration was measured using the latex agglutination method. Serum albumin concentration was measured by the bromocresol green albumin method. Serum CEA and CA19-9 concentrations were measured by an electrochemiluminescene immunoassay. Furthermore, therapeutic approach and the occurrence of cholangitis during cancer treatment were recorded. Treatment plans were determined by standard protocols according to the tumor stage and physical condition of the patient.

Statistical analysis. The patients in this study were divided into two groups according to 1-year survival, namely those surviving for $<1$ year ( $n=11,7$ men and 4 women) and those surviving for $\geq 1$ year ( $n=11,7$ men and 4 women). Means \pm standard deviation in both groups were calculated for continuous variables: Age, BMI, smoking habit (pack-years), number of teeth present, mean PPD, mean CAL, \%BOP, plaque level, and concentrations of HbA1c, CRP, albumin, CEA and CA19-9. Numbers and percentages are presented for categorical variables: Gender, cancer site, cancer stage, presence of chemotherapy, presence of cholangitis and severity of periodontitis. The Mann-Whitney U test and Fisher's exact test were used to assess significant differences in clinical variables between the two groups. Median survival was estimated using the Kaplan-Meier method and differences were tested using the log-rank test. The follow-up period was 1 year after diagnosis. Backward stepwise regression procedures for the Cox proportional hazards model were used to evaluate significant prognostic factors. In the Cox proportional hazards model, the following 7 candidate variables were selected: Age, gender, BMI, serum concentration of HbA1c, CA19-9 and $\mathrm{CRP}$, and severity of periodontitis. $\mathrm{P}<0.05$ was considered to indicate statistically significant differences. All the analyses were performed using the SPSS 15.0 J software program for Windows (IBM Japan, Tokyo, Japan).

\section{Results}

Patient characteristics. The characteristics of all the patients are summarized in Table I. Significant differences between the $<1$-year and the $\geq 1$-year survival groups were observed in serum CRP concentration $(\mathrm{P}=0.013)$, serum CEA concentration $(\mathrm{P}=0.028)$, mean $\mathrm{CAL}(\mathrm{P}=0.031)$ and severity of periodontitis $(\mathrm{P}=0.033)$. There were no significant differences in the other variables between the two groups.

Multivariate analysis of prognostic factors. Kaplan-Meier analyses using log-rank tests revealed that BMI, serum CRP concentration and severity of periodontitis were significant prognostic factors for survival rate (Fig. 1). However, the Cox proportional hazards regression model demonstrated that serum CA19-9 and CRP concentrations were significantly associated with the prognosis of pancreatobiliary tract cancer (Table II).

Comparison of CRP, albumin and HbAlc according to the severity of periodontitis. The serum CRP concentration was significantly higher and the serum albumin concentration was significantly lower in patients with severe periodontitis group when compared to those without severe periodontitis $(\mathrm{P}<0.05$; Table III). 
Table I. Differences in clinical parameters between the $<1$-year and $\geq 1$-year survival groups.

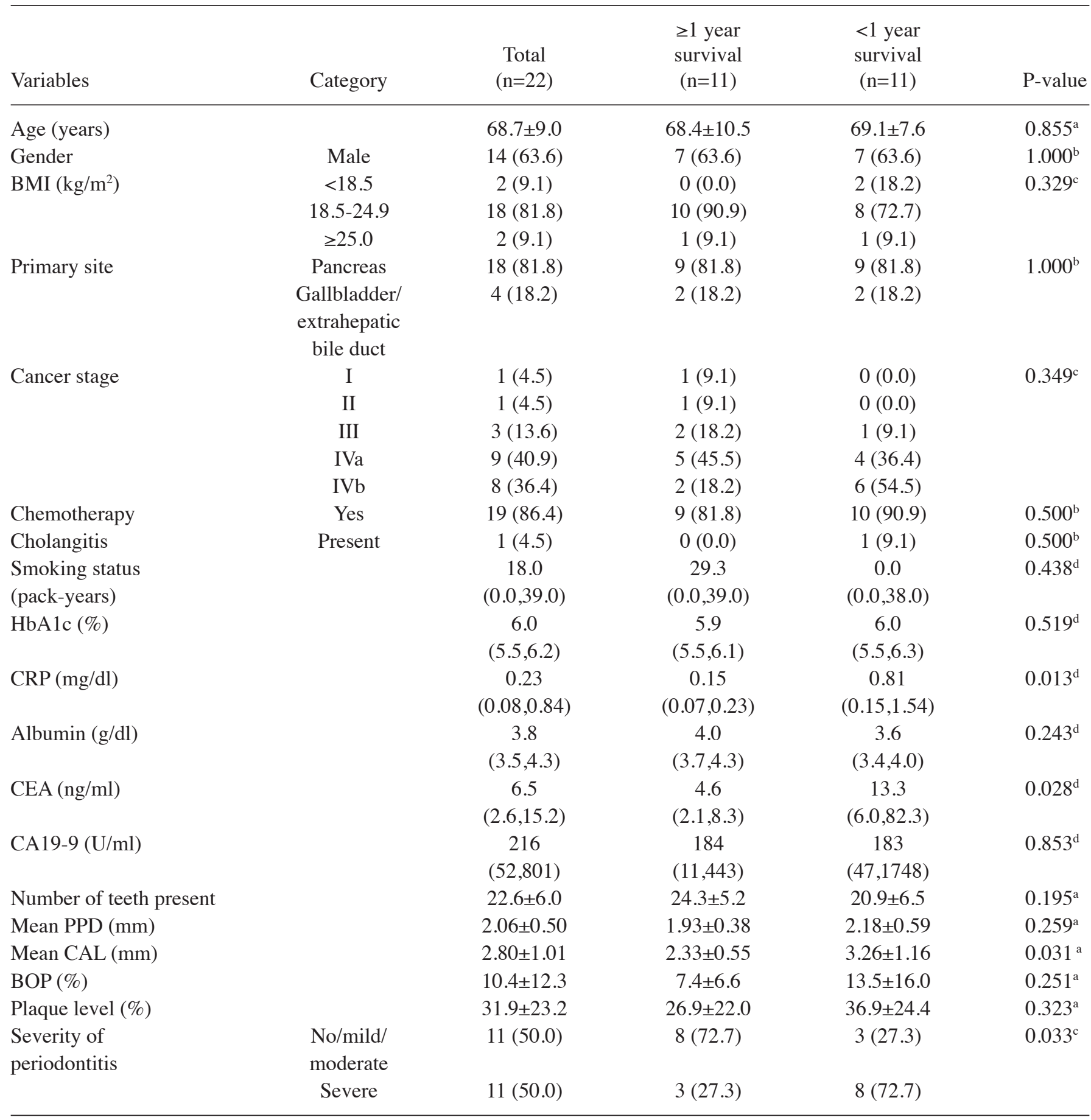

Values are presented as $\mathrm{n}(\%)$, mean \pm standard deviation, or median (25th, 75 th percentile). ${ }^{a}$ Unpaired t-test. ${ }^{b}$ Fisher's exact test. ${ }^{c} \mathrm{Chi}$-squared test. ${ }^{\mathrm{d}}$ Mann-Whitney U test. BMI, body mass index; HbA1c, hemoglobin A1c; CRP, C-reactive protein; CEA, carcinoembryonic antigen; CA19-9, carbohydrate antigen 19-9; PPD, probing pocket depth; CAL, clinical attachment level; BOP, bleeding on probing.

\section{Discussion}

To the best of our knowledge, this is the first study to investigate the association between the severity of periodontitis and the prognosis of pancreatobiliary tract cancer. In this study, the $<1$-year survival group exhibited higher levels of serum CRP concentration, serum CEA concentration, CAL and severity of periodontitis compared with the $\geq 1$-year survival group. In addition, the Kaplan-Meier method demonstrated that low BMI, high serum CRP concentration and severe periodontitis were significant prognostic factors for survival rate. These results indicate that the prognosis of pancreatobiliary tract cancer was associated with the severity of periodontitis, as well as other factors, such as CRP. However, the Cox proportional hazards regression model demonstrated that the serum CA19-9 and CRP concentrations were significant 
Table II. Multivariate analysis of prognostic factors.

\begin{tabular}{lccc}
\hline Factors & $\begin{array}{c}\text { Hazard } \\
\text { ratio }\end{array}$ & $95 \%$ CI & P-value $^{\mathrm{a}}$ \\
\hline Age & 0.87 & $0.75-1.00$ & 0.060 \\
HbA1c $(\%)$ & 1.96 & $0.96-3.98$ & 0.064 \\
CA19-9 $(\mathrm{U} / \mathrm{ml})$ & 1.002 & $1.000-1.004$ & 0.030 \\
CRP $(\mathrm{mg} / \mathrm{dl})$ & 2.57 & $1.15-5.74$ & 0.022 \\
\hline
\end{tabular}

${ }^{a}$ Cox proportional hazards model. CI, confidence interval; HbA1c, hemoglobin A1c; CA19-9, carbohydrate antigen 19-9; CRP, C-reactive protein.

Table III. Comparisons of CRP, albumin, and HbA1c according to severity of periodontitis.

\begin{tabular}{|c|c|c|c|}
\hline Variables & $\begin{array}{c}\mathrm{No} / \mathrm{mild} / \\
\text { moderate } \\
\text { periodontitis } \\
(\mathrm{n}=11)\end{array}$ & $\begin{array}{c}\text { Severe } \\
\text { periodontitis } \\
(\mathrm{n}=11)\end{array}$ & P-value ${ }^{a}$ \\
\hline CRP (mg/dl) & $0.12(0.07,0.34)$ & $0.54(0.15,1.54)$ & 0.034 \\
\hline Albumin $(\mathrm{g} / \mathrm{dl})$ & $4.0(3.8,4.3)$ & $3.5(3.3,3.7)$ & 0.016 \\
\hline HbA1c (\%) & $6.0(5.5,6.1)$ & $5.9(5.1,7.0)$ & 1.000 \\
\hline
\end{tabular}

${ }^{a}$ Mann-Whitney $U$ test.Values are presented as median (25th, 75th percentile). CRP, C-reactive protein; HbA1c, hemoglobin A1c.

prognostic factors, whereas the severity of periodontitis was not. Therefore, the association between the severity of periodontitis and the prognosis of pancreatobiliary tract cancer may be indirect.

Serum CRP, an indicator of systemic inflammation, is produced mainly by the liver in response to inflammation, infection and tissue damage (14). According to our findings, cancer patients with severe periodontitis had higher levels of serum CRP compared with those without severe periodontitis. This indicates that pancreatobiliary tract cancer patients with severe periodontitis had more severe systemic inflammation. A previous study demonstrated that the 1-year overall survival rate and median survival time in patients with higher CRP concentration $(>0.3 \mathrm{mg} / \mathrm{dl})$ were lower compared with those in the patients with lower CRP concentration $(\leq 0.3 \mathrm{mg} / \mathrm{dl})(15)$. This suggests that periodontitis affects the prognosis of pancreatobiliary tract cancer via the upregulation of systemic inflammation.

Studies have reported a significant association between periodontitis and serum CRP concentration. There is evidence that individuals with chronic periodontitis have elevated serum CRP concentrations when compared with periodontally healthy controls (16). It has also been reported that the presence of Porphyromonas gingivalis in subgingival plaque is significantly associated with raised serum CRP concentration (17). These observations are consistent with the present results, which indicate that severe periodontitis induces elevated serum CRP concentration.
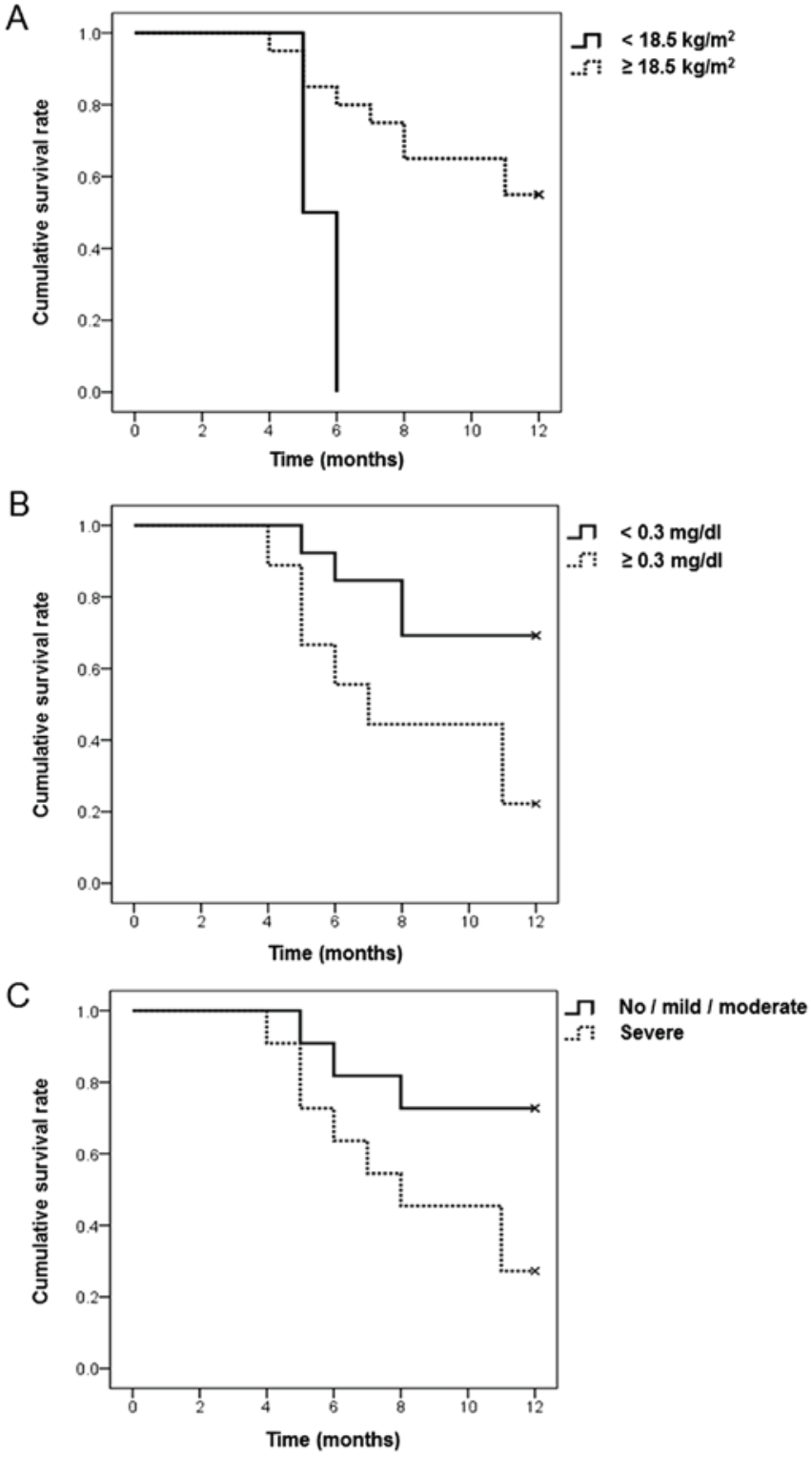

Figure 1. (A) Association between BMI ( $\left.<18.5 \mathrm{vs.} \geq 18.5 \mathrm{~kg} / \mathrm{m}^{2}\right)$ and overall survival in patients with pancreatobiliary tract cancer. The Kaplan-Meier analysis revealed significant differences between the two groups $(\mathrm{P}=0.019)$. (B) Association between CRP level ( $<0.3$ vs. $\geq 0.3 \mathrm{mg} / \mathrm{dl}$ ) and overall survival in patients with pancreatobiliary tract cancer. The Kaplan-Meier analysis revealed significant differences between the two groups $(\mathrm{P}=0.027)$. (C) Association between severity of periodontitis (no/mild/moderate vs. severe periodontitis) and overall survival in patients with pancreatobiliary tract cancer. The Kaplan-Meier analysis revealed significant differences between the two groups $(\mathrm{P}=0.045)$. BMI, body mass index; $\mathrm{CRP}, \mathrm{C}$-reactive protein.

Serum albumin is also known to be an independent predictor of survival in pancreatobiliary tract cancer (18). In our findings, serum albumin concentration was significantly lower in patients with severe periodontitis compared with that in patients without severe periodontitis. This suggests that periodontitis affects the prognosis of pancreatobiliary tract cancer by decreasing serum albumin level. However, there were no significant differences between the early and late survivor groups in terms of serum albumin concentration in this study. In the present study, the median serum albumin concentrations of the early and the late survivor group were 3.6 and $4.0 \mathrm{~g} / \mathrm{dl}$, respectively. As serum 
albumin concentration in this study was close to the reference value (3.8-5.3 g/dl), it may have little effect on the prognosis of pancreatobiliary tract cancer.

Several studies have reported a significant association between periodontitis and pancreatic cancer. Chang et al (11) reported that periodontal disease was positively associated with pancreatic cancer risk among patients aged $\geq 65$ years. Another study reported that individuals with high levels of antibodies against Porphyromonas gingivalis ATTC 53978 had a two-fold higher risk of pancreatic cancer compared with individuals with lower levels of these antibodies (19). Our results are supported by these findings indicating an association between periodontitis and pancreatic cancer. However, further studies are required to elucidate the mechanisms through which periodontitis affects the prognosis of pancreatic cancer.

There are few studies in the scale of a pilot study, although it is recommended to obtain $10 \%$ of the final study size (20). In the present study, the number of subjects in the $<1$-year and $\geq 1$-year survival groups was 11 each. When the final study size was calculated using the data of the current pilot study (Table I), 18 patients per group would have to be included to demonstrate significant differences between cancer patients with and those without severe periodontitis. As the present sample size was $>10 \%$ of the final study size, it is conceivable that 11 patients per group is sufficient as a pilot study.

The present study had certain limitations. First, all the patients were recruited from the Okayama University Hospital. This may limit the application of our findings to the general population. Second, the application of periodontal treatment during cancer treatment was not investigated in this study, which may have affected the prognosis of pancreatobiliary tract cancer.

In conclusion, pancreatobiliary tract cancer patients with severe periodontitis had a poorer prognosis compared with those without severe periodontitis. Severe periodontitis may be indirectly associated with the survival rate of pancreatobiliary tract cancer through promoting systemic inflammation.

\section{Acknowledgements}

This study was supported by a Grant-in-Aid for Scientific Research (26670904) from the Ministry of Education, Culture, Sports, Science and Technology, Tokyo, Japan.

\section{References}

1. Torre LA, Bray F, Siegel RL, Ferlay J, Lortet-Tieulent J and Jemal A: Global cancer statistics, 2012. CA Cancer J Clin 65: 87-108, 2015 .
2. Hundal R and Shaffer EA: Gallbladder cancer: Epidemiology and outcome. Clin Epidemiol 6: 99-109, 2014.

3. American Cancer Society. Cancer Facts \& Figures 2013. Atlanta, American Cancer Society, 2013.

4. Adami HO and Trichopoulos D: Pancreatic cancer. In: Ekbom A and Trichopoulos D (eds). Textbook of Cancer Epidemiology. 2nd edition. Oxford University Press, New York, pp333-348, 2008.

5. Fitzpatrick SG and Katz J: The association between periodontal disease and cancer: A review of literature. J Dent 38: 83-95, 2010

6. Joshipura KJ, Wand HC, Merchant AT and Rimm EB: Periodontal disease and biomarkers related to cardiovascular disease. J Dent Res 83: 151-155, 2004.

7. Loos BG: Systemic markers of inflammation in periodontitis. J Periodontol 76 (11 Suppl): S2106-S2115, 2005.

8. Amabile N, Susini G, Pettenati-Soubayroux I, Bonello L, Gil JM, Argues S, Bonfil JJ and Paganelli F: Severity of periodontal disease correlates to inflammatory systemic status and independently predicts the presence and angiographic extent of stable coronary artery disease. J Intern Med 263: 644-652, 2008.

9. Roshani R, McCarthy F and Hagemann T: Inflammatory cytokines in human pancreatic cancer. Cancer Lett 345: 157-163, 2014.

10. Michaud DS, Joshipura K, Giovannucci E and Fuchs CS: A prospective study of periodontal disease and pancreatic cancer in US male health professionals. J Natl Cancer Inst 99: 171-175, 2007.

11. Chang JS, Tsai CR, Chen LT and Shan YS: Investigating the association between periodontal disease and risk of pancreatic cancer. Pancreas 45: 134-141, 2016.

12. Eke PI, Page RC, Wei L, Thornton-Evans G and Genco RJ: Update of the case definitions for population-based surveillance of periodontitis. J Periodontol 83: 1449-1454, 2012.

13. Le N, Sund M and Vinci A; GEMS collaborating group of Pancreas 2000: Prognostic and predictive markers in pancreatic adenocarcinoma. Dig Liver Dis 48: 223-230, 2016.

14. Pepys MB and Hirschfield GM: C-reactive protein: A critical update. J Clin Invest 111: 1805-1812, 2003.

15. Kishi T, Nakamura A, Itasaka S, Shibuya K, Matsumoto S, Kanai M, Kodama Y, Takaori K, Mizowaki T and Hiraoka M: Pretreatment C-reactive protein level predicts outcome and patterns of failure after chemodadiotherapy for locally advanced pancreatic cancer. Pancreatology 15: 694-700, 2015.

16. Paraskevas S, Huizinga JD and Loos BG: A systematic review and meta-analyses on C-reactive protein in relation to periodontitis. J Clin Periodontol 35: 277-290, 2008.

17. Winning L, Patterson CC, Cullen KM, Stevenson KA, Lundy FT, Kee F and Linden GJ: The association between subgingival periodontal pathogens and systemic inflammation. J Clin Periodontol 42: 799-806, 2015.

18. Siddiqui A, Heinzerling J, Livingston EH and Huerta S: Predictors of early mortality in veteran patients with pancreatic cancer. Am J Surg 194: 362-366, 2007.

19. Michaud DS, Izard J, Wilhelm-Benartzi CS, You DH, Grote VA, Tjønneland A, Dahm CC, Overvad K, Jenab M, Fedirko V, et al: Plasma antibodies to oral bacteria and risk of pancreatic cancer in a large European prospective cohort study. Gut 62: 1764-1770, 2013.

20. Lackey NR and Wingate AL: The pilot study: One key to research success. In: Brink PJ and Wood MJ (eds). Advanced Design in Nursing Research. 2nd edition. Sage Publications, CA, 1998. 